Chapter 29

\title{
Regenerative Medicine for Neurological Diseases with the Use of Electrical Stimulation
}

\author{
Masahiro Kameda \\ Additional information is available at the end of the chapter \\ http://dx.doi.org/10.5772/55612
}

\section{Introduction}

In current clinical practice, tissue plasminogen activator (t-PA) is the gold standard drug to be administred within three hours after the onset of cerebral infarction. In the near future, it will become acceptable to administer it within 4.5 hours of onset. Even if t-PA administration cannot be utilized for cerebral infaction patients because of time window limitations, the intravascular technique using the Merci ${ }^{(R)}$ retreiver and the Penumbra ${ }^{(R)}$ system can be applied to some patients with cerebral infarction. Not all cerebral infarction patients can be saved by these clinical therapeutic methods. Despite surviving the acute phase of cerebral infarction as a result of these clinical therapeutic methods, some patients suffer from permanent hemiparesis in the chronic phase. It is for this reason that regenerative medicine must play more important role in solving this problem.

We have two types of regenerative medicine approaches to patients with neurological diseases such as cerebral infarction and Parkinson's disease. The first approach involves exogenous stem cells for stem cell transplantation [1-3], and the second involves the enhancement of endogenous stem cells. In terms of neural stem cell transplantation (allogenic transplantation), we demonstrated the neuroprotective effect of adult neural stem and progenitor cells that were modified to secrete Glial cell line-derived neurotropic factor (GDNF) in a transient ischemia model of rats [4]. However, we have not demonstrated the same effect by autologous transplantaion. Stem cells were transplanted in the acute stage of an ischemia animal model in many studies, which showed the neuroprotective effect of stem cell transplantation. It is difficult to show the same effect of stem cell transplantation in the chronic phase of ischemia. As a result, if we want to show the same effect by autologous stem cell transplantation, we must transplant autologous stem cells in an acute-stage ischea model of rats. Unfortunately, we do not yet have a technique to immediately expand autologous adult neural stem cells. Our previous experi- 
ment showed that it took several weeks to obtain a sufficient number of autologous adult neural stem cells [5]. Many researchers have pointed out that the effect of these stem cell transplantations is derived not from cell replacement, which is the original purpose of stem cell transplantation, but mainly from the trophic effect brought about by transplanted stem cell secretions. Generally speaking, compared to exogenous stem cell transplantation, the enhancement of endogenous stem cells is expected to be less invasive. Previous reports showed that an enriched environment and running enhanced endogenous stem cell generation [6] and that trophic factors produced by these enhanced endogenous stem cells had a neuroprotective effect on these neurological disorders. Deep brain stimulation (DBS) is one of the gold standards of treatment for Parkinson's disease (PD) in current clinical pratice. Recent reports have showed that DBS can enhance endogenous stem cells in a PD model of rats [7]. Although phase 3 or 4 clinical trials with DBS for depression and obesity and epidural electrical stimulation for pain are currently ongoing, they are not being performed for regenerative medicine (http://clinicaltrials.gov). Based on this report, we examined whether electrical stimulation has therapeutic potential for CNS diseases by activating endogenous stem cells.

\section{Electrical stimulation for cerebral infarction}

After the onset of cerebral infarction, more endgenous stem cells are produced in the SVZ and these endogenous stem cells migrate and differentiate into neurons in order to replace the area of infarction. Most of these newly produced endogenous stem cells cannot survive, however, and die within a few weeks [8]. That is why we expected that enhancing the survival of these newly produced endogenous stem cells or enhancing the production of endogenous stem cells by electrical stimulation could result in an improved neurorescue effect.

The invasiveness of the electrical stimulation of the brain varies with the position of the electrode, e.g., brain parenchyma, brain surface (subdural), epidural space, or skin. In the DBS for Parkinson's disease, electrodes are inserted in deep brain areas such as the subthalamic nucleus. Although DBS is considered to be one of the gold standards of therapy for Parkinson's disease, less invasive and more effective stimulation techniques are urgently required. Compared to the use of exogenous stem cells such as stem cell transplantation, the enhancement of endogenous stem cells is expected to be less invasive. We believe that continuous epidural stimulation is the best choice as it is less invasive and can be expected to produce a similar neuroprotective effect as DBS.

To examine the neurorescue effect of electrical stimulation for cerebral infarction, we applied epidural electrical stimulation to an acute-stage cerebral infarction model of rats. To identify the best stimulation parameter, we stimulated the rats with different frequencies and intensities (see Table). We found that continous electrical stimulation with low frequency produced a better neurorescue effect compared to that with high frequency. Moreover, this lowfrequency continous epidural electrical stimulation had a better neurorescue effect through an increase in trophic factors such as BDNF, GDNF, and VEGF (Fig.1), suppressed inflamatory response (Fig. 2), enhanced angiogenesis (Fig.2) and played anti-apoptotic effect with the 
upregulation of phosphorylated Akt (Fig.3). LY294002 administration into the lateral ventricle suppressed the neurorescue effect of electrical stimulation (Fig.4), which showed that this antiapoptotic effect was exerted through the PI3K-Akt signaling pathway [9].

\begin{tabular}{rrrrrl}
\hline & \multicolumn{1}{c}{0} & \multicolumn{1}{c}{2} & \multicolumn{1}{c}{10} & \multicolumn{1}{c}{50} & (Hz) \\
\hline 100 & $3.9 \pm 1.5$ & $7.6 \pm 3.2$ & $8.2 \pm 2.2$ & $4.4 \pm 1.7$ & (LPT at 3 days) \\
& $6.3 \pm 2.7$ & $11.8 \pm 2.2$ & $11.1 \pm 3.2$ & $6.2 \pm 0.9$ & (LPT at 1 week) \\
& $28.0 \pm 1.5$ & $21.3 \pm 1.4$ & $21.8 \pm 6.9$ & $30.5 \pm 8.7$ & (infarct volume) \\
200 & & $8.0 \pm 2.7$ & & & (LPT at 3 days) \\
& & $10.3 \pm 3.8$ & & & (LPT at 1 week) \\
$(\mu \mathrm{A})$ & & $23.9 \pm 8.2$ & & & (infarct volume) \\
\hline
\end{tabular}

Table 1. The table presents Limb Placement Test (LTP) scores (3 days and 1 week after MCAO) and the percentages of infarct volumes measured 3 days post-MCAO relative to the intact side of rats receiving electrical stimulation with several parameters. Initially, 0, 2, 10, and $50 \mathrm{~Hz}$ were used with $100 \mu \mathrm{A}$ current. Next, exploration using $200 \mu \mathrm{A}$ was performed. Based on this experiment, we decided that $2 \mathrm{~Hz} 100 \mu \mathrm{A}$ stimulaton was the optimal therapeutic condition. This table was reproduced and/or modified from the original article [9].

\section{A}

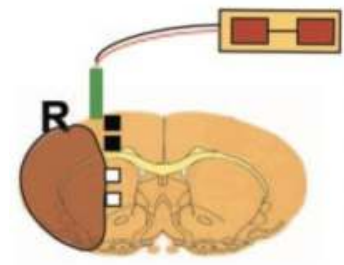

B
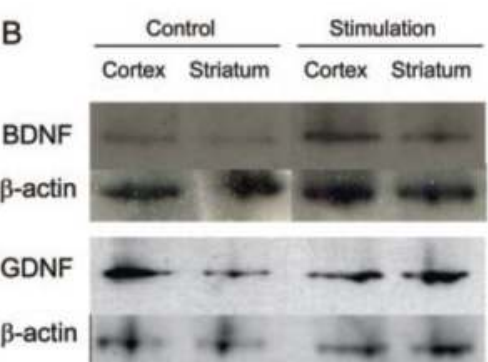

VEGF

$\beta$-actin
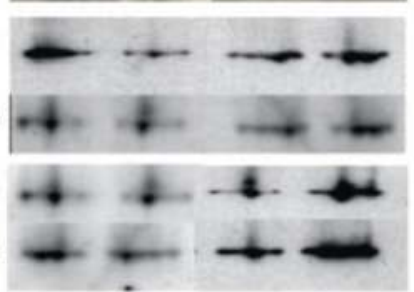

Control cortex

Stinulation cortex
Control striatum

Stimulation striatum
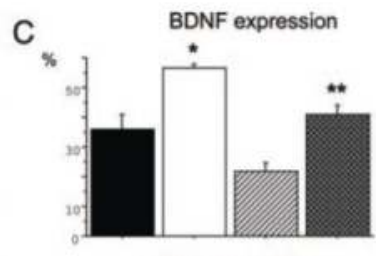

$\%$

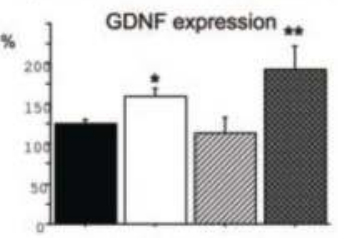

VEGF expression

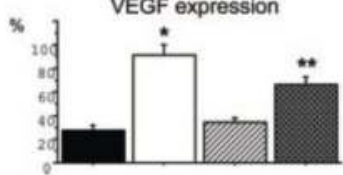

Figure 1. (A) The schematic diagram shows the regions in the cortex and striatum of rats with electric stimulation. Black box, cortex; white box, striatum. Each pair of brain tissues in the cortex and striatum per hemisphere was punched. (B) Upregulation of neurotrophic factors such as BDNF, GDNF, and VEGF was observed in the stroke rats receiving electric stimulation. (C) Quantification. ${ }^{*} p<0.05$ versus cortex of the control rats. ${ }^{* *} p<0.05$ versus striatum of the control rats. This figure was reproduced and/or modified from the original article [9]. 

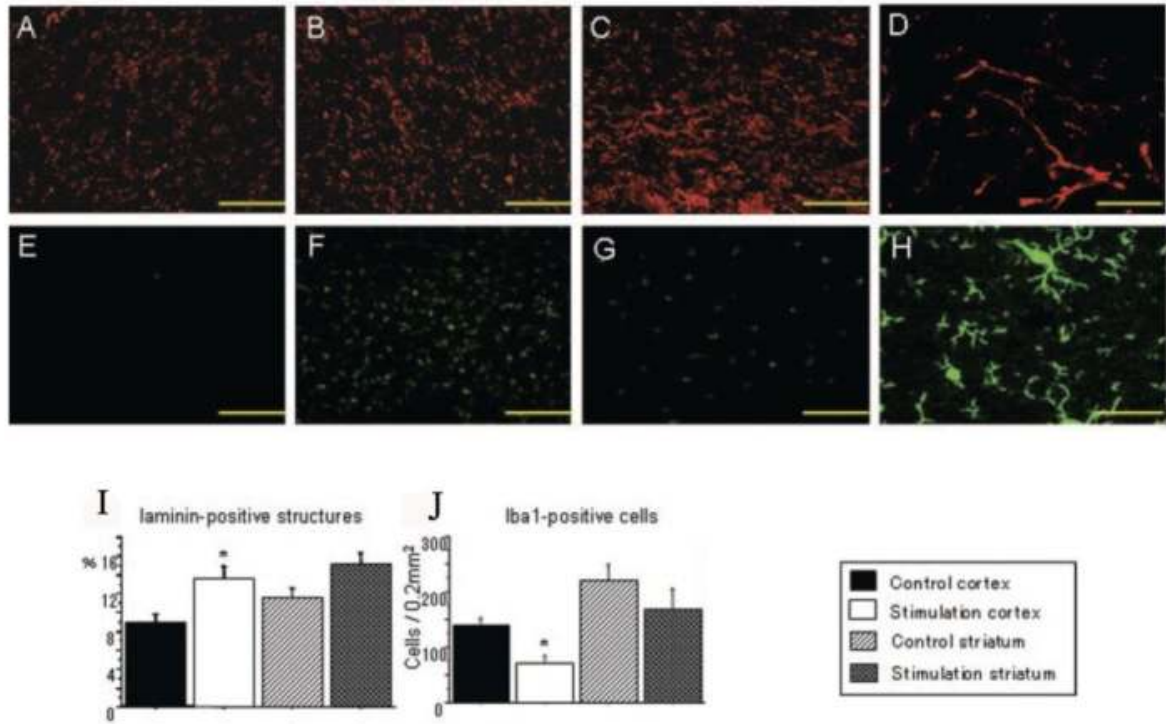

Figure 2. Laminin staining ( $A-D$ and $I$ ) and Iba-1 staining ( $E-H$ and $J)$ show angiogenic and anti-inflammatory effects in the stimulation cortex were exerted by electric stimulation. A and $\mathrm{E}$ are the intact cortex, $\mathrm{B}$ and $\mathrm{F}$ are nonstimulated cortex, and $C, D, G$, and $H$ are the ischemic cortex ( $D$ and $H$ are with high magnification). * $p<0.05$ versus cortex of the control rats (I and J). Scale bar: $100 \mu \mathrm{m}(\mathrm{A}-\mathrm{C}, \mathrm{E}-\mathrm{G}), 25 \mu \mathrm{m}(\mathrm{D}, \mathrm{H})$. This figure was reproduced and/or modified from the original article [9].
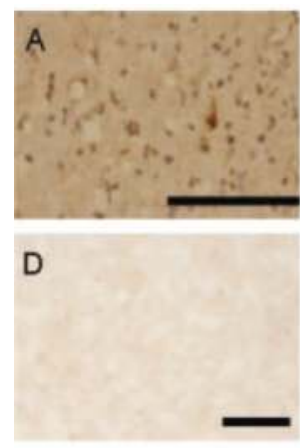

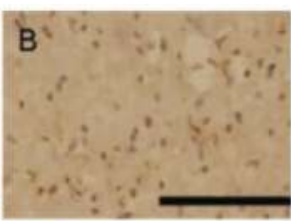

E
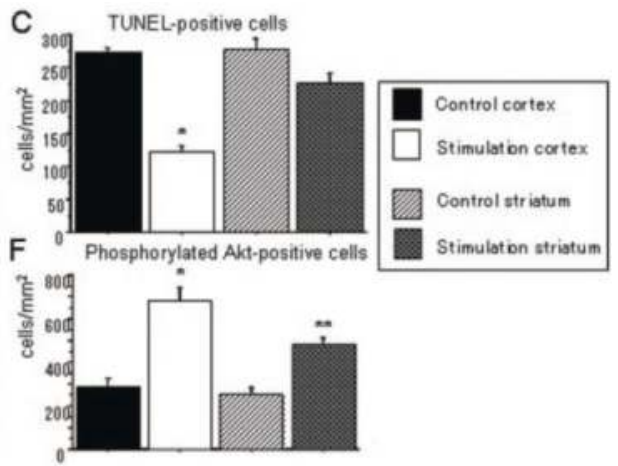

Figure 3. Anti-apoptotic effects of electric stimulation were exerted through phosphorylated Akt. (A-C): TUNEL staining revealed the anti-apoptotic effect of electrical stimulation (B) compared to those in the control group (A). Scale bar: $100 \mu \mathrm{m}$. (C): Quantitative analyses. ${ }^{*} p<0.05$ versus cortex of the control rats. (D-F): Phosphorylated Akt staining revealed a surge of stained cells in the ischemic cortex with electric stimulation (E) compared with the control group (D). (F) Quantitative analyses. ${ }^{*} p<0.05$ versus cortex of the control rats. ${ }^{* *} p<0.05$ versus striatum of the control rats. This figure was reproduced and/or modified from the original article [9]. 

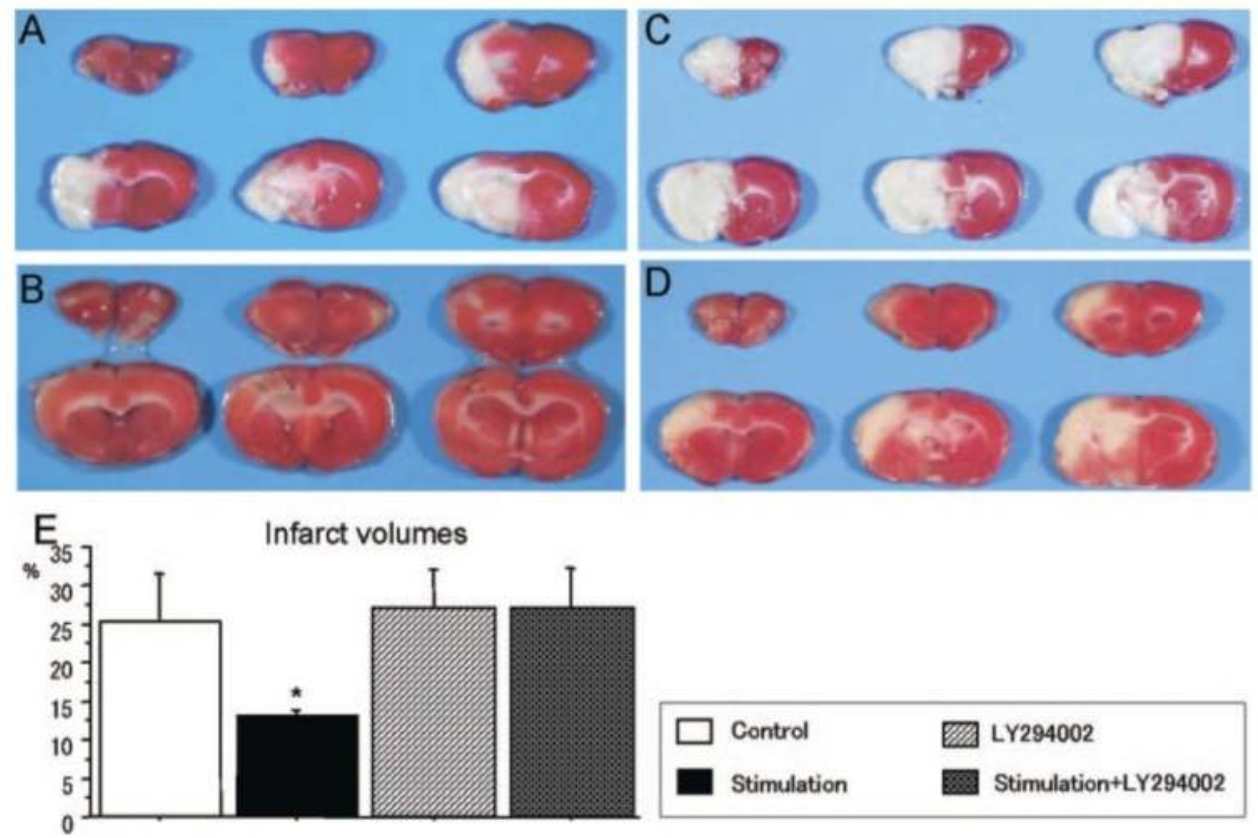

$\begin{array}{ll}\square \text { Control } & \text { LY294002 } \\ \text { Stimulation } & \text { Stimulation+LY294002 }\end{array}$

Figure 4. LY294002 blocked the neuroprotective effects of electric stimulation. The infarct volumes of rats receiving electric stimulation (B) were significantly decreased compared with those of control rats (A) and ischemic rats that received LY294002 alone (C). In contrast, the neuroprotective effects of electric stimulation were blocked by intraventricular administration of LY294002 (D). Quantitative analysies of infarct volumes is shown in E. * $p<0.05$ versus all other groups. This figure was reproduced and/or modified from the original article [9].

As the next step, we applied epidural electrical stimulation to a chronic-phase cerebral infarction model of rats. However, we could not obtain the same neuroprotective effect. This is why we needed to change the method. According to the clinical application of DBS for Parkinson's disease, we decided to insert an electrode in the infarct striatum although this method is more invasive than epidural stimulation. Before giving striatum electrical stimulation to the chronic-phase ischemia model of rats, we applied electrical stimulation to normal rats and found that low-frequency continuous striatal electrical stimulation upregulates GDNF and vascular endothelial growth factor (VEGF) and enhances the neuronal differnetiation of endogenous stem cells and angiogenesis. We then applied striatal stimulation to the chronicphase ischemia model of rats. We conducted magnetic resonance imaging 28 days after ischemic induction and inserted electrodes into the striatum based on these MR images followed by electrical stimualation for a week; rats were sacrificed on day 60 . In this experiment, we found that striatum stimulation exerted behavioral improvement and MR images taken after striatal stimulation showed a reduced infarct volume compared with those taken before stimulation. Moreover, striatal stimulation during the chronic-phase ischemia model of rats resulted in enhanced migration of neural progenitor cells from SVZ to ischemic 
penumbra, enhanced neuronal differentiation, and suppressed microglial activation in the ischemic penumbra [10].

\section{Long-Term Potentiation (LTP) and neurogenesis}

It is well established that new neurons are produced continuously in the SVZ and the subgranular zone (SGZ) of the dentate gyrus (DG) during adulthood [11-13]. Given this fact, many researchers have focused their attention on discovering how to stimulate these resident precursors to enhance neurogenesis. Environmental enrichment and voluntary running [14] are now widely accepted ways to enhance neurogenesis.

Long-term potentiation (LTP) is induced by brief high-frequency tetanization of excitatory afferents and is observed as a long-lasting enhancement in the efficacy of synaptic transmission [15]. LTP is considered to be a cellular model of learning [16] and memory [17]. Moreover, LTP is speculated to be one possible mechanism of enhanced motor function recovery resulting from electrical stimulation to the cortex after stroke [18]. Based on the accumulated evidence of hippocampal neurogenesis, the relationship among hippocampus-related function, LTP, and neurogenesis has been examined [19-22]. For example, environmental enrichment and running enhanced hippocampus-related memory function as well as LTP. More importantly, electrophysiological reports have demonstrated that recently created neurons contribute to synaptic plasticity [23] and that LTP correlates with neural plasticity, compensating for ischemia-induced damage [24]. These findings support the hypothesis that enhancing neurogenesis may be useful in the restoration and repair of a damaged brain and emphasizes the importance of finding a more effective way to stimulate neurogenesis. As mentioned above, a number of reports are available that describe the relationship between neurogenesis and LTP but these mainly focus on neurogenesis-dependent changes in LTP [19, 21, 22]. Only a few reports have shown that LTP per se can enhance neurogenesis [25-27].

Neural activity was recently demonstrated to stimulate a large number of hippocampal latent precursors, including a self-renewing stem cell population that only becomes activated following depolarization [28]. Given a number of recent reports linking LTP and hippocampal neurogenesis, we examined whether LTP is able to enhance proliferation of the neural precursors and neurogenesis in the adult mouse dentate gyrus. In this study, we stimulated the perforant pathway unilaterally with several stimulation protocols and analyzed hippocampal precursor numbers using both the neurosphere assay and BrdU immunostaining. In the neuropshere assay, we found that induction of late-LTP, but not early-LTP, was required to activate latent precursors in the mouse dentate gyrus and that neurosphere number was positively correlated with LTP magnitude (Fig.5 \& Fig.6). In the BrdU immnostaining, proliferation and differentiation in the dentate gyrus was significantly enhanced in the only mice in which LTP was successfully induced. In contrast, protocols that failed to induce LTP, induced only early LTP; used low-frequency stimulation (LFS) or included a pharmacological blocker of LTP (CPP: (3-(2-carboxypiperazin-4-yl)propyl-1-phosphonic acid), all failed to activate neurogenesis (Fig.7). These findings demonstrate that LTP can activate latent neural precursor cells in the adult mouse dentate gyrus, leading to increased neurogenesis [29]. 


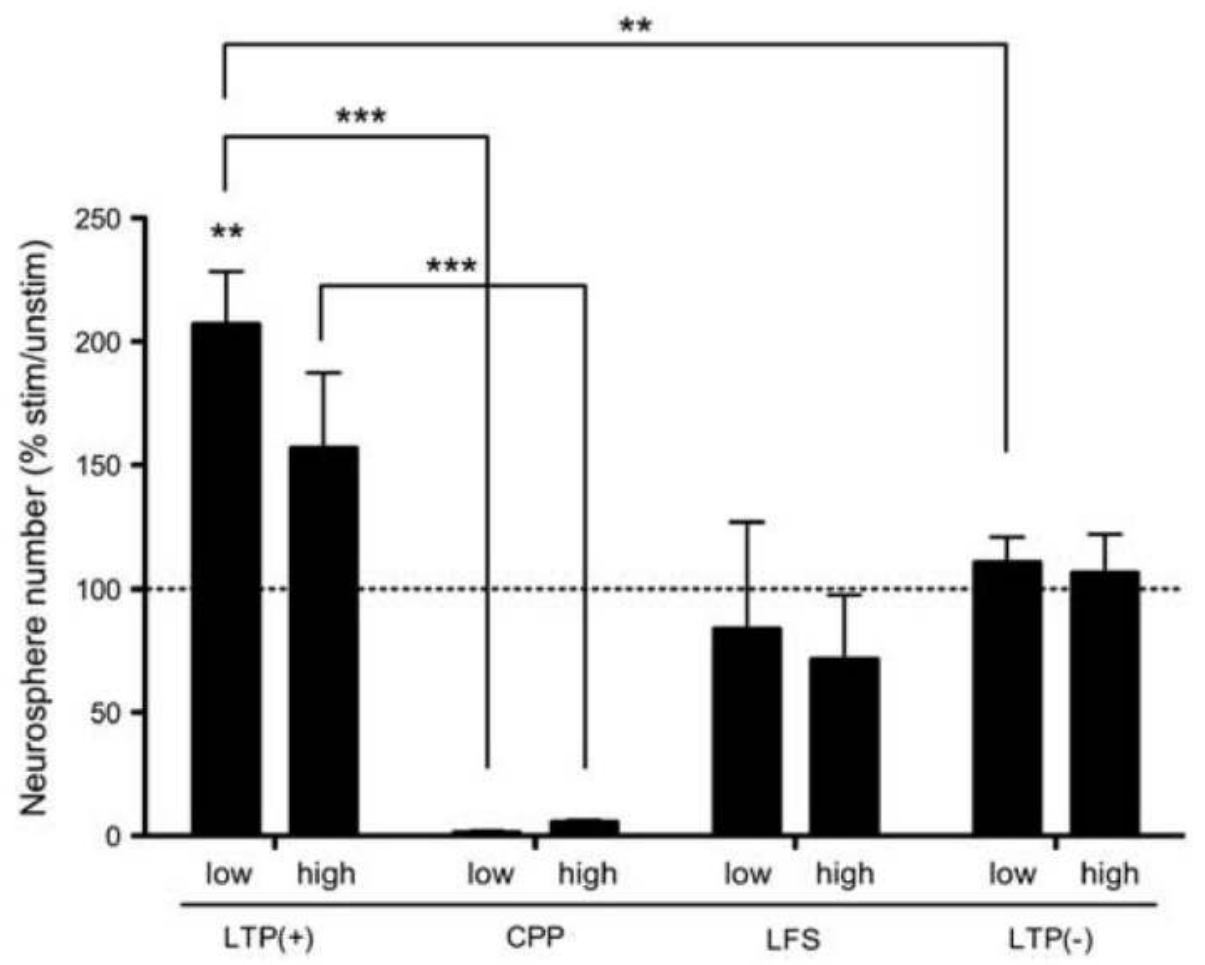

Figure 5. LTP enhances proliferation of neural precursors. A neurosphere formation assay was performed 2 days after stimulation. Under normal $\mathrm{K}^{+}$conditions, only the LTP $(+)$group revealed a significant increase in neurosphere number from the stimulated hemisphere relative to the control hemisphere. Furthermore, a significantly greater number of neurospheres was generated from the stimulated hippocampi of the LTP(+) group compared with the simulated hippocampi of the CPP group and LTP(-) group. When CPP was administered to block the NMDA receptor, neurosphere formation was significantly reduced compared with the LTP $(+)$ group. low: low $\mathrm{K}^{+}$, high: high $\mathrm{K}^{+} .{ }^{* *} p<0.01,{ }^{* \star *} p<$ 0.001. This figure was modified and/or reproduced from the original article [29]. 

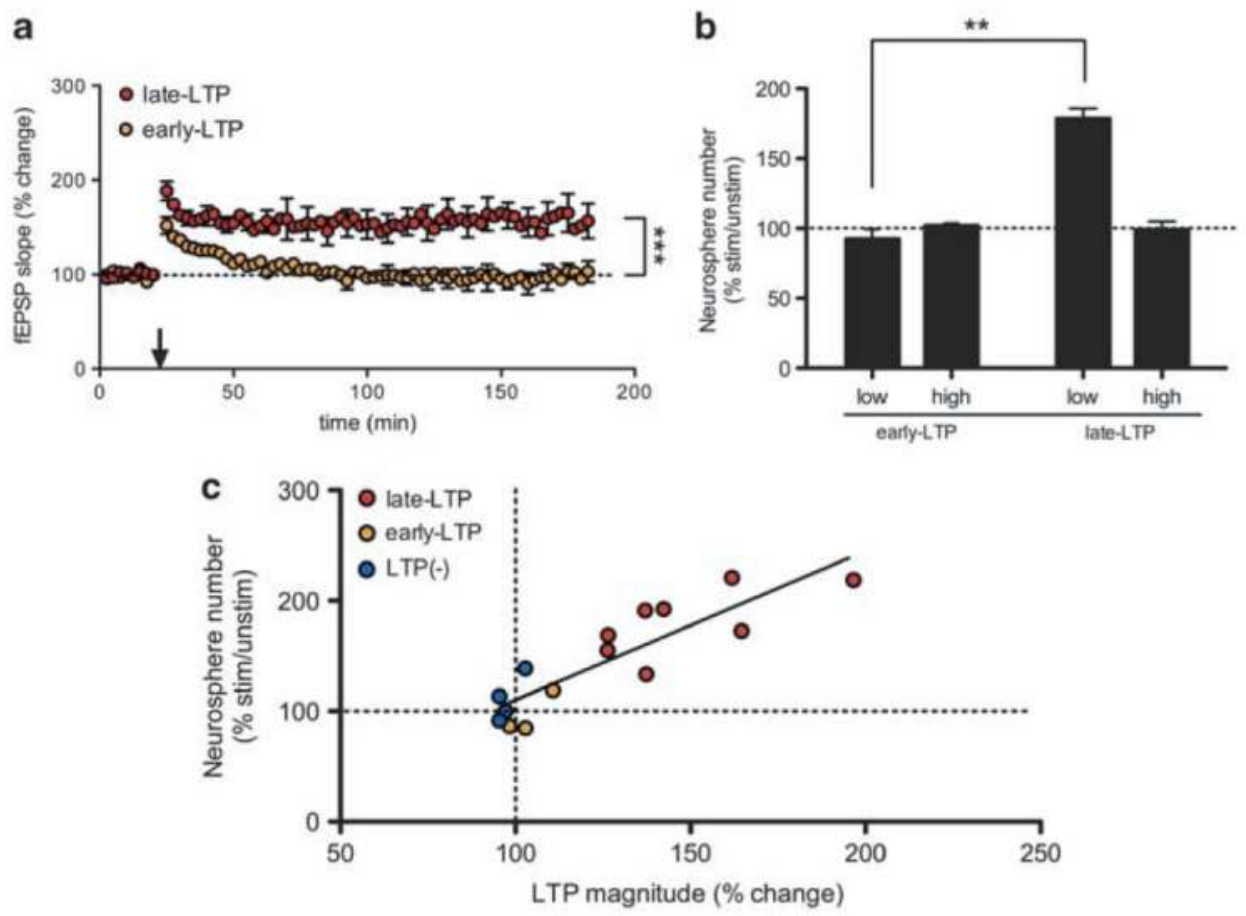

Figure 6. Late-LTP but not early-LTP can activate hippocampal precursors in vitro. (a) At the end of recording, the magnitude of potentiation in the late-LTP group was significantly greater than that in the early-LTP group. (b) Following the induction of late-LTP, a significant increase in the number of neurospheres was observed in the low $\mathrm{K}^{+}$condition compared with the early-LTP group. (c) The magnitude of LTP, calculated 60 min after HFS, showed a significant positive correlation ( $r=0.879, n=15, p<0.0001$, Pearson's correlation) with the number of neurospheres grown in vitro, after hippocampi were dissected 2 days after HFS. The arrow indicates HFS, error bars indicate SEM, low: low K+, high: high $\mathrm{K}^{+} .{ }^{* *} p<0.01,{ }^{* * *} p<0.001$. Abbreviations: fEPSP, field extracellular postsynaptic potential; HFS, high-frequency stimulation. This figure was modified and/or reproduced from the original article [29]. 

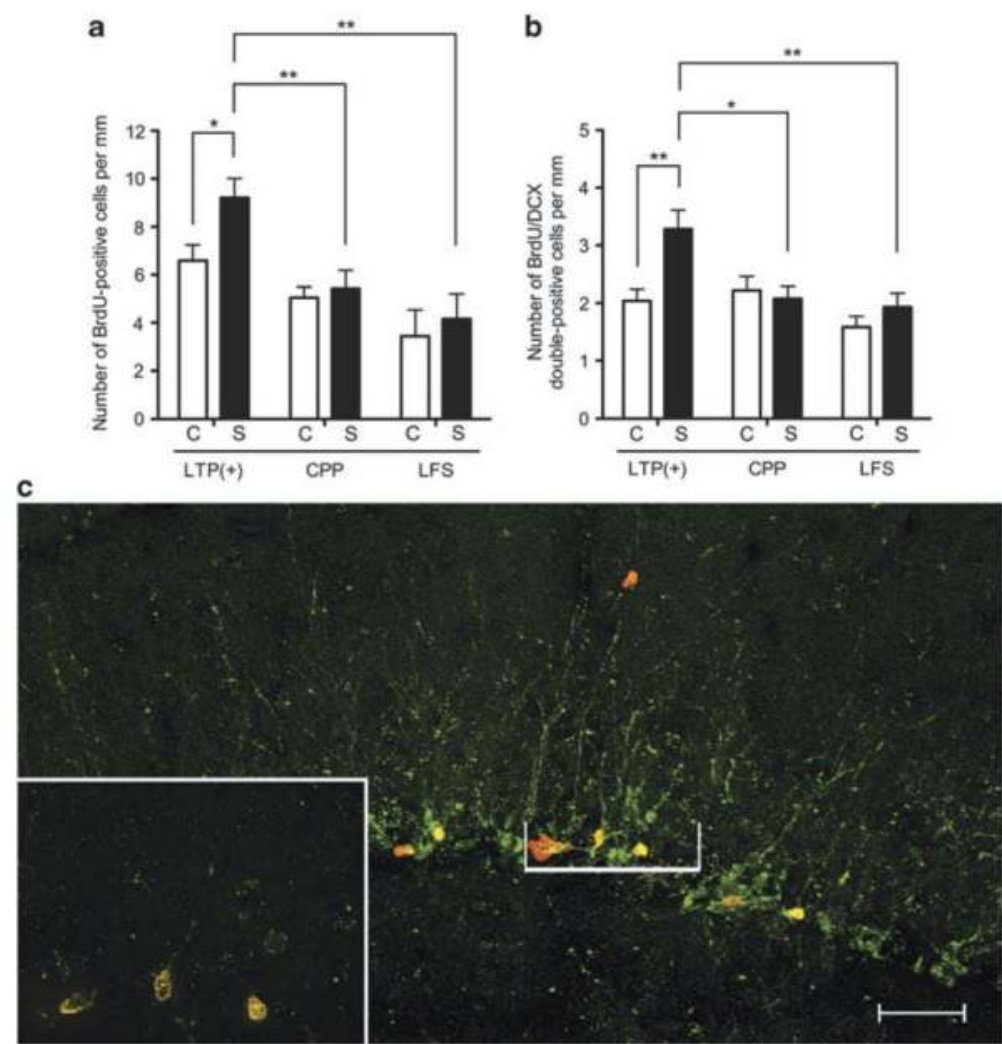

Figure 7. Proliferation and differentiation were significantly enhanced in the LTP(+)-stimulated hippocampus. (a) The number of BrdU-positive cells per length of the dentate gyrus $(\mathrm{mm})$ was significantly enhanced in stimulated hippocampi of the LTP(+) group compared to the unstimulated hemisphere. Furthermore, there was a greater number of BrdU-positive cells following LTP induction compared with the CPP group and LFS group. (b) Following LTP induction, the number of BrdU/DCX double-positive cells per perimeter length of the dentate gyrus (mm) was significantly enhanced in the stimulated hippocampus compared with the control hippocampus. BrdU/DCX-double labeling also revealed a significantly greater number of neurons in the stimulated hippocampi of the LTP(+) group compared with the stimulated hippocampi of the CPP and the LFS groups. (c) Representative maximum intensity projection image of BrdU (red) and DCX (green) immunostaining reconstructed from 40 optical sections of the LTP-stimulated hippocampus of the LTP(+) group. Inset shows a higher magnification. Scale bar: $50 \mathrm{~mm}$ in main image, $20 \mathrm{~mm}$ for inset. ${ }^{*} p<0.05,{ }^{* *} p$ $<0.01$. Abbreviations: C, control side; DCX, doublecortin; S, stimulated side. This figure was modified and/or reproduced from the original article [29].

In the future, these findings can be clinically applied to individuals suffering from depression, Alzheimer's disease, and age-related cognitive decline explained by neuronal loss. In fact, electrical convulsive seizure is clinically performed for severe depression and this therapy showed increase of the proliferation and neuronal differnetiation of hippocampal precursors in a depression model of animals [30-32]. For Alzheimer's disease, a previous report showed that transcranial direct current stimulation enhanced aspects of memory performance in both healthy controls and individuals with Alzheimer's disease [33]. However, an operation for 
electrode insertion is needed, and this is invasive for patients. To reduce invasiveness, transcranial magnetic stimulation (TMS), which is currently clinically applied to patients with severe depression, would solve this problem. For age-related cognitive decline, TMS is currently the main therapeutic option; however, more evidence needs to be collected. Continuous learning and exercise is the best method to overcome age-related cognitive decline.

In the future, we need to clarify the mechanism of electrical stimulation and neurogenesis in more detail. In the electrical stimulation conducted for cerebral infarction, we found that the optimal stimulation parameter was low frequency; however, in this LTP experiment, neurogenesis was enhanced by HFS that can induce LTP. Disease model (cerebral infarction) or healthy model is a crucial difference between these two experiments. This difference in animal condition might be one possible explanation for this discrepancy in optimal stimulation parameters. As written above, we found the therapeutic potentials of electrical stimulation; however, further studies and ethical consensus are required for its application in a clinical setting because electrical stimulation has the potential to modulate the human mind and cognition.

\section{Conclusion}

The therapeutic benefit of electrical stimulation to neurological diseases was reviewed in terms of the enhancement of endogenous stem cells. Electrical stimulation was demonstrated to play a neuroprotective and/or neurorescue effect in the ischemia model of animals. For intact animals, LTP is most effective at activating hippocampal latent stem cells. Electrical stimulation should be considered a possible therapeutic method in the future, although further studies and ethical consensus are required before use in the clinical setting.

\section{Acknowledgements}

The author appreciates Professor Perry Bartlett, Professor Cliff Abraham, Dr. Chanel Taylor, Dr. Tara Walker, and Dr. Yasuhara Takao for their valuable support. I am also grateful to Drs. Tanefumi Baba, Naoki Tajiri, Feifei Wang, and Takamasa Morimoto for their efforts and contributions.

\section{Author details}

\section{Masahiro Kameda}

Department of Neurological Surgery, Okayama University Graduate School of Medicine, Dentistry and Pharmaceutical Sciences, Japan 


\section{References}

[1] Tajiri, N, Yasuhara, T, Shingo, T, Kondo, A, Yuan, W, Kadota, T, Wang, F, Baba, T, Tayra, J. T, Morimoto, T, Jing, M, Kikuchi, Y, Kuramoto, S, Agari, T, Miyoshi, Y, Fujino, H, Obata, F, Takeda, I, Furuta, T, \& Date, I. (2010). Exercise exerts neuroprotective effects on Parkinson's disease model of rats. Brain Research, , 1310, 200-207.

[2] Muraoka, K, Shingo, T, Yasuhara, T, Kameda, M, Yuen, W. J, Uozumi, T, Matsui, T, Miyoshi, Y, \& Date, I. (2008). Comparison of the therapeutic potential of adult and embryonic neural precursor cells in a rat model of Parkinson disease. J Neurosurg, 108, , 149-159.

[3] Takahashi, K, Yasuhara, T, Shingo, T, Muraoka, K, Kameda, M, Takeuchi, A, Yano, A, Kurozumi, K, Agari, T, Miyoshi, Y, Kinugasa, K, \& Date, I. (2008). Embryonic neural stem cells transplanted in middle cerebral artery occlusion model of rats demonstrated potent therapeutic effects, compared to adult neural stem cells. Brain Research, 1234, , 172-182.

[4] Kameda, M, Shingo, T, Takahashi, K, Muraoka, K, Kurozumi, K, Yasuhara, T, Maruo, T, Tsuboi, T, Uozumi, T, Matsui, T, Miyoshi, Y, Hamada, H, \& Date, I. (2007). Adult neural stem and progenitor cells modified to secrete GDNF can protect, migrate and integrate after intracerebral transplantation in rats with transient forebrain ischemia. European Journal of Neuroscience, , 26, 1462-1478.

[5] Muraoka, K, Shingo, T, Yasuhara, T, Kameda, M, Yuan, W, Hayase, H, Matsui, T, Miyoshi, Y, \& Date, I. (2006). The high integration and differentiation potential of autologous neural stem cell transplantation compared with allogeneic transplantation in adult rat hippocampus. Experimental Neurology, 199, , 311-327.

[6] Gage, F. H. (2000). Mammalian neural stem cells. Science, 287, , 1433-1438.

[7] Toda, H, Hamani, C, Fawcett, A, Hutchison, W, \& Lozano, A. (2008). The regulation of adult rodent hippocampal neurogenesis by deep brain stimulation. J Neurosurg, 108, , 132-138.

[8] Lindvall, O, Kokaia, Z, \& Martinez-serrano, A. (2004). Stem cell therapy for human neurodegenerative disorders-how to make it work. Nat Med.;10 Suppl: S, 42-50.

[9] Baba, T, Kameda, M, Yasuhara, T, Morimoto, T, Kondo, A, Shingo, T, Tajiri, N, Wang, F, Miyoshi, Y, Borlongan, C. V, Matsumae, M, \& Date, I. (2009). Electrical Stimulation of the Cerebral Cortex Exerts Antiapoptotic, Angiogenic, and Anti-Inflammatory Effects in Ischemic Stroke Rats through Phosphoinositide 3-Kinase/Akt Signaling Pathway. Stroke, 40, , e598-e605.

[10] Morimoto, T, Yasuhara, T, Kameda, M, Baba, T, Kuramoto, S, Kondo, A, Takahashi, K, Tajiri, N, Wang, F, Meng, J, Ji, Y. W, Kadota, T, Maruo, T, Kinugasa, K, Miyoshi, Y, Shingo, T, Borlongan, C. V, \& Date, I. (2010). Striatal stimulation nurtures endoge- 
nous neurogenesis and angiogenesis in chronic-phase ischemic stroke rats. DOI:X544915

[11] Reynolds, B. A, \& Weiss, S. (1992). Generation of neurons and astrocytes from isolated cells of the adult mammalian central nervous system. Science 255, 1707-10.

[12] Richards, L. J, Kilpatrick, T. J, \& Bartlett, P. F. (1992). De novo generation of neuronal cells from the adult mouse brain. Proc Natl Acad Sci USA, 89, 8591-5.

[13] Gage, F. H, Coates, P. W, Palmer, T. D, Kuhn, H. G, Fisher, L. J, Suhonen, J. O, Peterson, D. A, Suhr, S. T, \& Ray, J. (1995). Survival and differentiation of adult neuronal progenitor cells transplanted to the adult brain. Proc Natl Acad Sci USA, 92, 11879-83.

[14] Blackmore, D. G, Golmohammadi, M. G, Large, B, Waters, M. J, \& Rietze, R. L. (2009). Exercise Increases Neural Stem Cell Number in a Growth Hormone-Dependent Manner, Augmenting the Regenerative Response in Aged Mice. Stem Cells , 27, 2044-52.

[15] Bliss, T. V, \& Collingridge, G. L. (1993). A synaptic model of memory: long-term potentiation in the hippocampus. Nature , 361, 31-9.

[16] Moser, E. I, Krobert, K. A, Moser, M. B, \& Morris, R. G. (1998). Impaired spatial learning after saturation of long-term potentiation. Science , 281, 2038-42.

[17] Martin, S. J, Grimwood, P. D, \& Morris, R. G. (2000). Synaptic plasticity and memory: an evaluation of the hypothesis. Annu Rev Neurosci , 23, 649-711.

[18] Teskey, G. C, Flynn, C, Goertzen, C. D, Monfils, M. H, \& Young, N. A. (2003). Cortical stimulation improves skilled forelimb use following a focal ischemic infarct in the rat. Neurol Res , 25, 794-800.

[19] Van Praag, H, Christie, B. R, Sejnowski, T. J, \& Gage, F. H. (1999b). Running enhances neurogenesis, learning, and long-term potentiation in mice. Proc Natl Acad Sci USA, 96, 13427-31.

[20] Duffy, S. N, Craddock, K. J, Abel, T, \& Nguyen, P. V. (2001). Environmental enrichment modifies the PKA-dependence of hippocampal LTP and improves hippocampus-dependent memory. Learn Mem , 8, 26-34.

[21] Farmer, J, Zhao, X, Van Praag, H, Wodtke, K, Gage, F. H, \& Christie, B. R. (2004). Effects of voluntary exercise on synaptic plasticity and gene expression in the dentate gyrus of adult male Sprague-Dawley rats in vivo. Neurosci 124, 71-9.

[22] Wang, J. W, David, D. J, Monckton, J. E, Battaglia, F, \& Hen, R. (2008). Chronic fluoxetine stimulates maturation and synaptic plasticity of adult-born hippocampal granule cells. J Neurosci , 28, 1374-84.

[23] Snyder, J. S, Kee, N, \& Wojtowicz, J. M. (2001). Effects of adult neurogenesis on synaptic plasticity in the rat dentate gyrus. J Neurophysiol , 85, 2423-31. 
[24] Wang, S, Kee, N, Preston, E, \& Wojtowicz, J. M. (2005). Electrophysiological correlates of neural plasticity compensating for ischemia-induced damage in the hippocampus. Exp Brain Res , 165, 250-60.

[25] Bruel-jungerman, E, Davis, S, Rampon, C, \& Laroche, S. (2006). Long-term potentiation enhances neurogenesis in the adult dentate gyrus. J Neurosci 26, 5888-93.

[26] Chun, S. K, Sun, W, \& Jung, M. W. (2009). LTD induction suppresses LTP-induced hippocampal adult neurogenesis. Neuroreport , 20(14), 1279-83.

[27] Chun, S. K, Sun, W, Park, J. J, \& Jung, M. W. (2006). Enhanced proliferation of progenitor cells following long-term potentiation induction in the rat dentate gyrus. Neurobiol Learn Mem , 86, 322-9.

[28] Walker, T. L, White, A, Black, D. M, Wallace, R. H, Sah, P, \& Bartlett, P. F. (2008). Latent stem and progenitor cells in the hippocampus are activated by neural excitation. J Neurosci , 28, 5240-7.

[29] Kameda, M, Taylor, C. J, Walker, T. L, Black, D. M, Abraham, W. C, \& Bartlett, P. F. (2012). Activation of latent precursors in the hippocampus is dependent on long-term potentiation. Transl Psychiatry 2:e72. doi:tp.2011.70.

[30] Malberg, J. E, Eisch, A. J, Nestler, E. J, \& Duman, R. S. Chronic antidepressant treatment increases neurogenesis in adult rat hippocampus. ((2000). J Neurosci; , 20, 9104-10.

[31] Madsen, T. M, Treschow, A, Bengzon, J, Bolwig, T. G, Lindvall, O, \& Tingström, A. Increased neurogenesis in a model of electroconvulsive therapy. ((2000). Biol Psychiatry; , 47, 1043-9.

[32] Scott, B. W, Wojtowicz, J. M, \& Burnham, W. M. Neurogenesis in the dentate gyrus of the rat following electroconvulsive shock seizures. ((2000). Exp Neurol; , 165, 231-6.

[33] Moreines, J. L, Mcclintock, S. M, \& Holtzheimer, P. E. Neuropsychologic effects of neuromodulation techniques for treatment-resistant depression: A review. ((2011). Brain Stimul; , 4, 17-27. 
